\title{
Subject Index, Vol. 28, 1996
}

N-Acetylcysteine 245, 270 Acute liver failure 402 Acute-phase proteins 96 Adenine nucleotides 212 Aminoguanidine 167 Anaesthesia, epidural 70 Anastomoses 26, 374 Animal model 227 Anterior gastric wall stapling 154 Antibiotic 351

- $\quad$ therapy 395 Arterial autograft 39

- ketone body ratio 212 Artificial matrix 32 Ascorbic acid 306 Asialofetuin 201

ATP 140

Autologous fibrin tissue adhesive 162

\section{Bacteria 351}

Bacterial translocation 341 , 402 Bacteriological techniques 124 Bile ducts 299

- secretion 146 Bilirubin 1 Bioassay 447 Biochemical stability 447 Biocompatibility 162 Blood gases 227

- glucose 70

Body composition 295 Bone induction 447

- marrow 227

- morphogenetic protein 447

C-reactive protein 190 Cancer 167

Capillary blood flow, experimental 395

- leak 167 Cathepsin 361 Cell adhesion 428

- origin 380

- seeding 49 Cholangitis 63

Cholecystokinin 299

Choledochoduodenostomy 63

Cholestasis 104

Cholesterol saturation 1

Chronic stimulation 80

Cirrhosis 413

Cisapride 402

Class I MHC expression 256

Cold ischemia, cirrhotic liver 111

Colectomy 1

Collagen 32,436

Colon 374

Colonic anastomoses 315

Common bile duct 32

- $\quad$ iliac artery 39 Complement 440 Cu 306 Cyclosporin 262

- A 39

Degradation, bone-inducing

activity 447 Denervation, microsurgical 367 Devazepide 299 Dextran 395

Disintegration, gallstones 179 DNA synthesis 212 Dog 419 Dysphagia 461

Endocytosis, receptor-mediated

201 Endogenous endotoxemia 341 Endothelial cell(s) 428, 440 Endothelin 14 Endothelium 49, 380 Endotoxemia 323

Endotoxin(s) 55,130,351 Endotoxin-neutralizing capacity

130 Energy charge 212

- expenditure 295

Esophageal carcinoma 26

Esophagus 461

Ethanol precipitation 162

Experimental peritonitis 124, 287

Fast, enteral 146 Fat embolism 227 Fe 306 
Fibrin sealant 436 Fibrinolysis 287 Fibrinolytic potential 380 5-Fluorouracil 374 Folinicacid 374 Formaldehyde-treated bovine serum albumin 201

Galactose elimination capacity 333

- tolerance test 333

Gallstones 179

Gastrectomy 1

Gastric mucosal damage 278 Gene engineering 440 Glutathione, reduced and oxidized form 245

Heart, working 388 Hemodynamics, pig 8 -, pulmonary 227 Heparin-like glycosaminoglycan

428 Hepatectomy 341,402 Hepatic arterial buffer response

323

- blood flow 323

- functional reserve 333

- microcirculation 270

- parenchymal ratio 333 Hepatocellular integrity 245 Hepatocyte(s) 201,222,256

- growth factor 413 Histamine 278 Histology 162

HPLC gel filtration 447 Human recombinant tissue-type

plasminogen activator 287 Hyaluronic acid 111 Hydatid disease 466 Hyperbaric oxygen tensions 235 Hypotension 70

Hypoxemia 227

474

IL-6 transgenic mouse 361 Imipenem 395 Immunogenicity 447 Indium-lll-oxine 49 Infection 395 Intact cell hypothesis 333

Interleukin-1 278 Interleukin-13 55 Interleukin-2 167 Interleukin-4 119 Interleukin-6 55, 96, 190, 278,

361 Intestinal ischemia 14

- permeability 341 Intimal hyperplasia 39 Intracystic application 466 Intraperitoneal abscesses 179

- loss of gallstones 179 Ionized calcium 1 Ischemia 75 Ischemia-reperfusion injury, cold

245 Isolated limb perfusion 235

- perfusion model, rat liver 111

Isovolemic hemodilution 395

Jaundice, obstructive 201

Laparoscopic cholecystectomy 179

- surgery 124

Laparoscopy 8

Latissimus dorsi 80

LDH 80

Leukocyte adhesion receptors 190 Leukocytes 428 Liposomes 140 Liver 299

- atrophy 212

- circulation 323

- cirrhosis 333

- - cold ischemia tolerance

- oxygenation 419

- regeneration 212,413

- resection 8, 104

- surgery 436

- transplantation 419 Lung carcinoma 461

- metastasis 167 Lymphokine-activated killer cells

167

Manometry 461 Mebendazole 466 Melanoma 235 Melphalan 235 Mesenteric circulation 14 Mg 306 Micrograft 39

Mitochondrial oxidases $80 \mathrm{Mn} 306$

Monoclonal antibodies 55 Motility 402,461 Muscle degradation 361

- transformation 80

Myosine 80

Necrosis 395 Nitric oxide 167,323 Nucleation time 1 Nutrition, parenteral 146

Obesity, surgery 295 Obstructive jaundice 333 Omentoplasty 315 Organ failure 130 
Pancreas 367 Pancreatic function 154 Pancreatitis, acute 395 Pantothenic acid 306 Partial hepatectomy 96, 413 Peritoneal adhesions 315

- cavity 124

Peritonitis 119,351

Pharmacokinetics 287

Phosphodiesterase inhibitor 388

Physiopathology 70

Plasma 130

Plasminogen activator inhibitor

287,380 Pneumonectomy 461 Pneumoperitoneum 8 Portal vein ligation 212

- $\quad$ - stenosis 341

- venous flow 146

- $\quad \mathrm{PCO}_{2} 146$

Posterior truncal vagotomy 154

Predictor 419

Primary closure, common bile

duct 32 Pringle maneuver 8 Prostacyclin analog 14 Prosthesis, blood vessel 49 Pulmonary air leak 162

Rat(s) 75,222,256,315,402 Regeneration, hepatic 262 Reperfusion 75, 140

- injury 270

Resected liver volume 96

Retroviral vector 440

Sarcoma 235

Scar 306

Segmental normothermic liver ischemia 256

Sepsis 323

Shock 140

Sick cell hypothesis 333

Silver staining, nucleolar organizing regions 63

Sinusoidal endothelial cell 201

- $\quad$ oxidative stress 245

Skeletal muscle function 75

Small bowel 222

Sodium nitroprusside 323

Somatostatin 262

Stomata 124

Stunning 388

Subcutaneous oxygen tension 235

Surgery 26, 190

T cells 167

Taurolidine 351

Tissue oxygen tension 26

- pH 235

- plasminogen activator 380 Trace elements 306 Transplantation 222 Trophic effects 299

Tumor necrosis factor 119, 278, 351

- - - alpha 55

- $\mathrm{pH} 235$

Vascular occlusion 104 Verapamil 75

Whole-blood stimulation 55 Wound healing 306, 374

Xenotransplantation 440

Zn 306

Subject Index Vol. 28, 1996

475 